\title{
Vol. 69, No. 11
}

In the report "Initial Investigation of Transmission of COVID-19 Among Crew Members During Quarantine of a Cruise Ship - Yokohama, Japan, February 2020," on page 312 , the second sentence of the first complete paragraph in the second column should have read "The earliest laboratoryconfirmed COVID-19 cases in crew members occurred in food service workers; 15 of the 20 confirmed cases in crew members occurred among food service workers who prepared food for other crew members and passengers, and 16 of the 20 cases occurred among persons with cabins on deck 3 , the deck on which the food service workers lived (Table)." 